\title{
Photopolymer Diffractive Optical Elements in Electronic Speckle Pattern Shearing Interferometry
}

\author{
Emilia Mihaylova \\ Technological University Dublin, emilia.mihaylova@tudublin.ie \\ Izabela Naydenova \\ Technological University Dublin, izabela.naydenova@tudublin.ie \\ Barry Duignan \\ Technological University of Dublin, Barry.Duignan@tudublin.ie
}

See next page for additional authors

Follow this and additional works at: https://arrow.tudublin.ie/cieoart

Part of the Other Engineering Commons

\section{Recommended Citation}

Mihaylova, E. et al. (2006) Photopolymer Diffractive Optical Elements in Electronic Speckle Pattern Shearing Interferometry. Optics and Lasers in Engineering, Vol. 44, no. 9, pp. 965-974. doi:10.1016/ j.optlaseng.2005.06.017

This Article is brought to you for free and open access by the Centre for Industrial and Engineering Optics at ARROW@TU Dublin. It has been accepted for inclusion in Articles by an authorized administrator of ARROW@TU Dublin. For more information, please contact arrow.admin@tudublin.ie, aisling.coyne@tudublin.ie, gerard.connolly@tudublin.ie.

Funder: Technological Sector Research Programme Strand III supported by the Irish Government.

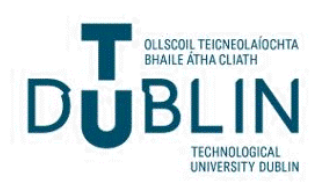




\section{Authors}

Emilia Mihaylova, Izabela Naydenova, Barry Duignan, Suzanne Martin, and Vincent Toal 


\title{
Photopolymer Diffractive Optical Elements in Electronic Speckle Pattern Shearing Interferometry
}

\author{
Emilia Mihaylova, Izabela Naydenova, Barry Duignan ${ }^{1}$, Suzanne Martin, Vincent Toal \\ Centre for Industrial and Engineering Optics \\ Dublin Institute of Technology, Kevin Street, Dublin 8, Ireland \\ e-mail: emilia.mihaylova@dit.ie \\ ${ }^{1}$ Faculty of Engineering, Dublin Institute of Technology \\ Bolton Street, Dublin 1, Ireland
}

\begin{abstract}
In this paper we present an electronic speckle pattern shearing interferometer using a photopolymer diffractive optical element in the form of a holographic grating, in combination with a ground glass to shear the images. The sheared images on the ground glass are further imaged onto a CCD camera. The distance between the grating and the ground glass can be used to control the shear and to vary the sensitivity of the system. The direction of sensitivity is easily controlled by rotation of the diffraction grating around its normal.

Introducing photopolymer holographic gratings in ESPSI gives the advantage of using high aperture optical elements at relatively low cost. The fact that the diffractive optical element is a photopolymer layer on glass substrate with thickness of $2 \mathrm{~mm}$ makes for a compact optical system.

The system was successfully used for detection of the resonant frequencies of a vibrating object.

Most of the published work on vibration analysis is analytical. Very few experimental results are available in the literature. The well known laser Doppler vibrometers (LDV) and accelerometers used for modal analysis are pointwise measurement techniques, although multipoint LDV is available at significant cost.

Electronic speckle pattern techniques suitable for experimental detection of the resonant frequencies of vibrating objects are very promising for vibration analysis because they are whole field and non contact.

A finite element model is developed for prediction of the vibration modes of the object under test. Detection of vibrational modes of aluminium diaphragm is demonstrated and compared with the theoretical model. The results obtained are very promising for future application of ESPSI systems with HOEs, for modal analysis. A significant advantage of shearography over electronic speckle pattern interferometry is that ESPSI is relatively insensitive to external disturbances. Another advantage of the proposed system is that it could be easily converted to a phase-shifting electronic speckle shearing interferometer.
\end{abstract}

KEYWORDS: ESPSI, shearing interferometry, holographic gratings, modal analysis, vibrations, FEA, phase-shifting 


\section{INTRODUCTION}

Electronic speckle pattern shearing interferometry (ESPSI) is an optical technique that enables full-field and non-contact direct measurements of displacement derivatives to be made [1-8]. A common method for generating two sheared images of the object in ESPSI systems is to use a Michelson interferometric optical set-up. The shear is introduced and controlled by tilting one of the mirrors.

The idea of using a holographic grating for shearing of the two images in speckle shearing interferometry is not new. Some early non-electronic systems used photographic gratings [5-7], but the use of CCD cameras enables observation of real-time fringe formation [8, 9]. Recently there has been an increasing interest in the application of shearography for modal analysis of vibrating objects. New interferometric systems are of interest for engineering and industrial applications.

We present the use of photopolymer holographic grating in a ESPSI system for modal analysis. Self-processing acrylamide based photopolymer [10] is used as a recording medium for recording holographic gratings. The optimized photopolymer material gives good diffraction efficiencies up to $94 \%$ for an exposure of $80 \mathrm{~mJ} / \mathrm{cm}^{2}$ and it performs well in the transmission mode of hologram recording. The holographic grating is used in combination of a ground glass to shear the images and to control the size of the shear.

\section{THEORY}

When two light waves interfere, the following equation [11] relates their relative phase $\Phi$ at a point to their relative geometrical path difference $L$ :

$$
\Phi=\frac{2 \pi}{\lambda} n L-\beta
$$

where $\lambda$ is the wavelength of the laser light, $n$ is the refractive index of the medium through which the laser light is transmitted, and $\beta$ is a constant phase. The change in the relative phase $\Delta=\delta \Phi$ or phase change, which manifests as visible fringes, can be effected by an incremental change in any of the three parameters $\lambda$, $n$, and $L$. Thus,

$$
\Delta=\frac{\partial \Phi}{\partial \lambda} \delta \lambda+\frac{\partial \Phi}{\partial n} \delta n+\frac{\partial \Phi}{\partial L} \delta L=-\frac{2 \pi L n}{\lambda^{2}} \delta \lambda+\frac{2 \pi L}{\lambda} \delta n+\frac{2 \pi n}{\lambda} \delta L
$$

where $\delta \lambda, \delta n$, and $\delta L$ denote respectively, the incremental change in wavelength, in refractive index and the difference in relative geometrical path length of the interfering waves. 
If the same wavelength is used and the test environment is still air, only the $\delta L$ term in Eq. (2) is nonzero, resulting in the following equation for the phase change [12]:

$$
\Delta=\frac{2 \pi}{\lambda}[A \delta u+B \delta v+C \delta w]
$$

where $u, v$ and $w$ are the displacement components of the point $P^{\prime}(x+\Delta x, y+\Delta y, z+\Delta z)$ relative to point $P(x, y, z)$ on the test surface, and $A, B$, and $C$ are sensitivity factors that are related to the optical arrangement. When a diffraction grating is used to introduce the shear the direction in which the system is sensitive can be precisely controlled by changing the orientation of the grating. If the grating introduces small image shearing $\Delta x$ (Fig. 1 a), the displacement terms in Eq. (3) can be expressed in terms of partial derivatives:

$$
\Delta=\frac{2 \pi}{\lambda}\left[A \frac{\partial u}{\partial x}+B \frac{\partial v}{\partial x}+C \frac{\partial w}{\partial x}\right] \Delta x
$$

If the introduced shear is in the y direction (Fig. 1 b) the phase difference is:

$$
\Delta=\frac{2 \pi}{\lambda}\left[A \frac{\partial u}{\partial y}+B \frac{\partial v}{\partial y}+C \frac{\partial w}{\partial y}\right] \Delta y
$$

In the most general case, when the grating is oriented as shown in Fig. $1 \mathrm{c}$

$$
\Delta=\frac{2 \pi}{\lambda}\left[A \frac{\partial u}{\partial s}+B \frac{\partial v}{\partial s}+C \frac{\partial w}{\partial s}\right] \Delta s
$$

Where $\Delta s$ is the amount of shear in direction s, determined by the angle $\alpha$ (Fig. $1 \mathrm{c}$ ).

a)
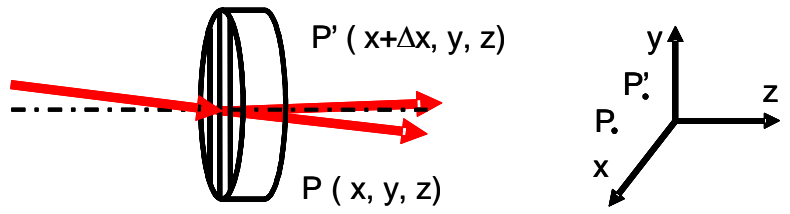

b)
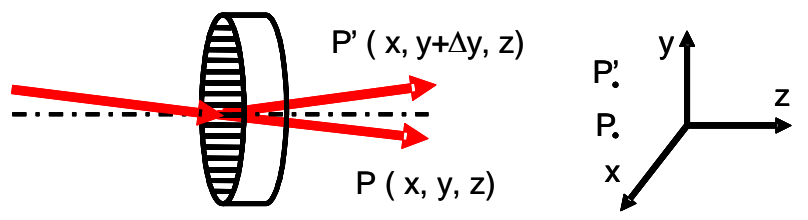

c)
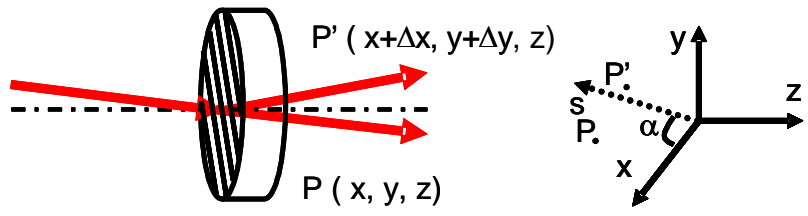

Fig. 1. Simple control of the shearing direction by rotation of the holographic diffraction grating. 
In our case (Fig. 2) the object beam lies in the (x, z) plane so there is no sensitivity in the y direction. In addition the grating orientation was as shown in Fig. 1a. The phase change is:

$$
\Delta=\frac{2 \pi}{\lambda}\left[A \frac{\partial u}{\partial x}+C \frac{\partial w}{\partial x}\right] \Delta x
$$

Consider the situation of an ESPSI system with one holographic grating in front of the CCD camera and small image shear $\Delta x$. The phase difference $\Delta$ can be expressed as [8]:

$$
\Delta=\frac{2 \pi}{\lambda}\left[\frac{\partial u}{\partial x} \sin \theta+\frac{\partial w}{\partial x}(1+\cos \theta)\right] \Delta x
$$

The dark fringes correspond to $\Delta=2 n \pi$, where $n$ is the fringe order. In this case:

$$
\frac{\partial u}{\partial x} \sin \theta+\frac{\partial w}{\partial x}(1+\cos \theta)=\frac{\lambda n}{\Delta x}
$$

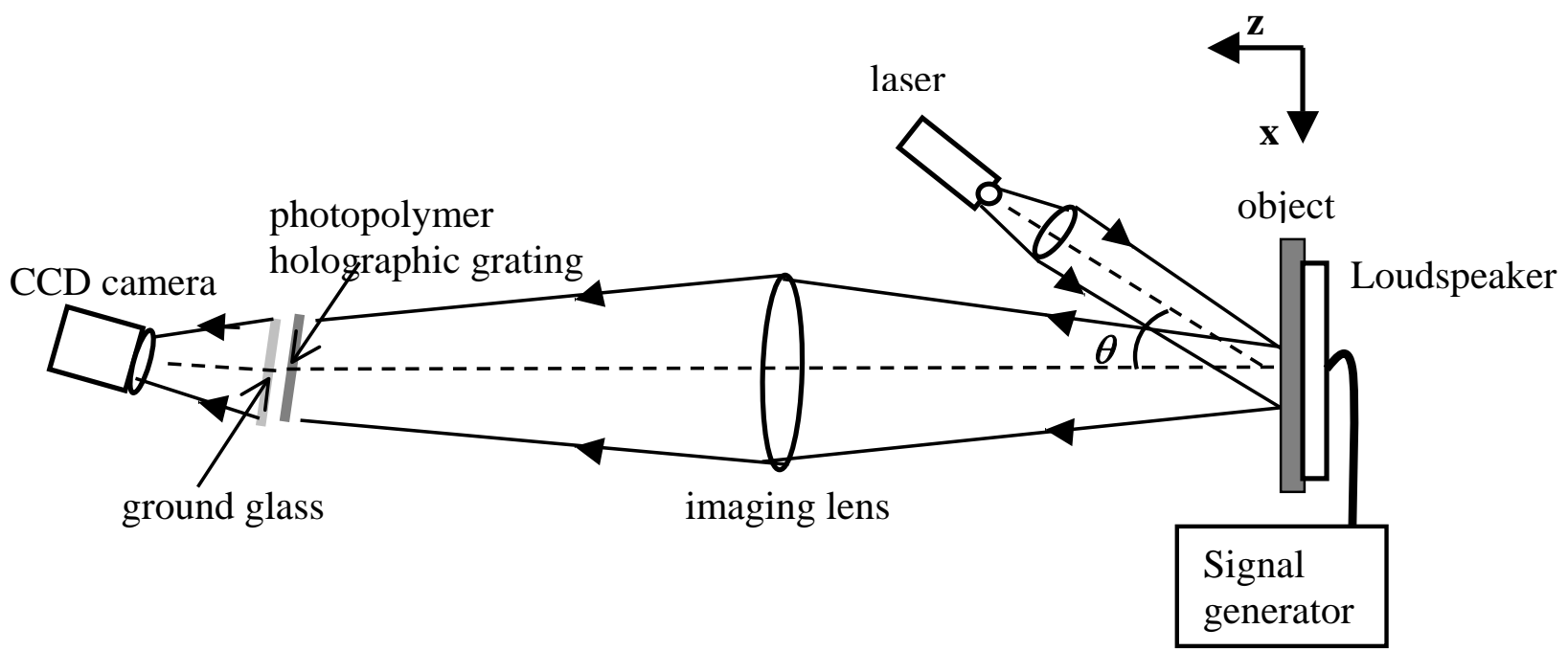

Fig.2. ESPSI system for vibration measurements with one photopolymer grating 


\section{EXPERIMENT}

\section{Photopolymer diffractive optical element}

Holographic gratings with spatial frequencies of 200 lines/mm and 500 lines/mm were recorded using the second harmonic of a CW $\mathrm{NdYVO}_{4}$ laser $(\lambda=532 \mathrm{~nm})$. The IEO acrylamide based photopolymer, which is self-developing, was used as the photosensitive medium. The layers were $100 \mu \mathrm{m}$ thick. Recording time and intensity were 20s and $3.5 \mathrm{~mW} / \mathrm{cm}^{2}$ respectively. The diameter of these gratings is $40 \mathrm{~mm}$. Diffraction efficiency in the +1 order is $60 \%$. One of the advantages of this material is that characteristics such as diffraction efficiency, thickness (which controls selectivity), slant angle, diameter and reconstruction wavelength and angle can all be chosen to suit the specific application. The IEO photopolymer is characterized by low scattering and this is important when the imaging properties of the optical system are of concern.

\section{Experimental set-up}

The scheme of the ESPSI system with a single photopolymer holographic grating is presented in Fig. 2. A similar system had been developed in our laboratory for static measurements and is described in detail elsewhere [13]. A Helium-Neon laser, with wavelength $633 \mathrm{~nm}$ and output power of $20 \mathrm{~mW}$, is used as the light source. The laser beam illuminates the object at an angle $\theta=30^{\circ}$ to the normal to the object surface. A lens images the object onto a ground glass, which acts as a diffusing screen. A holographic photopolymer diffraction grating is placed in front of the ground glass. The intensities of the zero and the first order of diffraction were equalized by rotation of the grating. The rotation of the grating around the central axis, parallel to y-axis, leads to slight off-Bragg angular adjustment and decrease in the intensity of the first order thus offering the possibility for fine adjustment of both image and sheared image intensities.

\section{Control of the shearing amount and shearing direction}

Easy control of the shearing amount is important because it determines the overall sensitivity of the system, see Eq. 9. The shearing amount can be controlled by changing the relative position of the ground glass and the diffraction grating as shown in Fig. 1. The increase of the distance between the two elements leads to increase of the amount of shear and increase of the sensitivity of the system. When the distance between the two elements is kept constant the shearing amount can be changed by utilizing diffraction gratings with different spatial frequencies. The higher is the spatial frequency the bigger is the amount of the shear. The spatial frequency of the grating influences also other important parameters of the system such as the contrast of the obtained ESPSI fringes and the size of the field of view. They will be discussed in the experimental section.

The rotation of the grating around its normal leads to a change in the shearing direction and thus controls the shearing direction and the direction of sensitivity.

The ESPSI system using HOE to shear the image is easy to apply in a phase-shifting mode. Mounting the glass plate or the HOE on a piezoelectric transducer (PZT) allows the introduction of a known phase shift. 


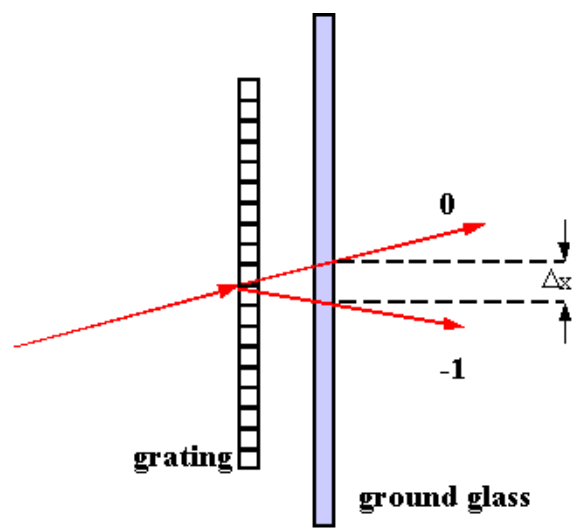

ground glass

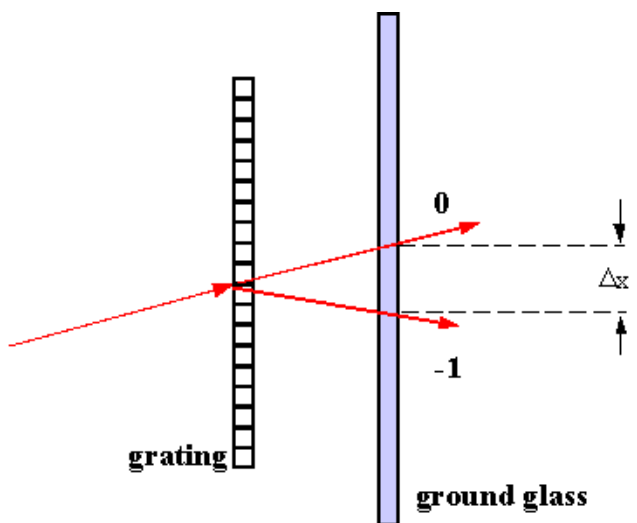

a

b

Fig. 3. Control of the shear

\section{Influence of the spatial frequency of the grating on the ESPSI fringes contrast and the size of the region under study}

In order to study the influence of the spatial frequency of the gratings on the quality of the obtained ESPSI fringes diffraction gratings with spatial frequencies of 200 and $500 \mathrm{l} / \mathrm{mm}$ were used. The system was evaluated by conducting two sets of experiments using a simple three point bending test, one with each grating. The shear was varied from $1 \mathrm{~mm}$ to $12 \mathrm{~mm}$ simply by varying the distance between the photopolymer holographic grating and the ground glass (Fig. 3).

It was observed that the ESPSI fringes obtained with the grating having spatial frequency $500 \mathrm{lines} / \mathrm{mm}$ have better contrast. When the spatial frequency of the grating is low (Fig. 4a), due to its lower angular selectivity, the diffraction orders -1 and or +2 are visible in addition to the main orders 0 and +1 .

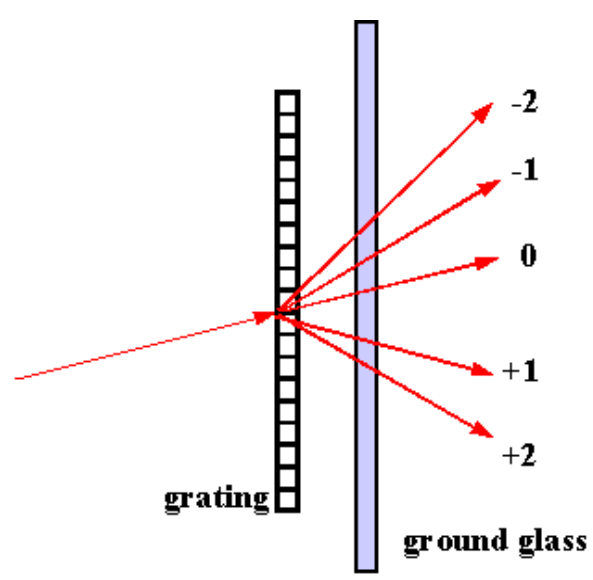

a

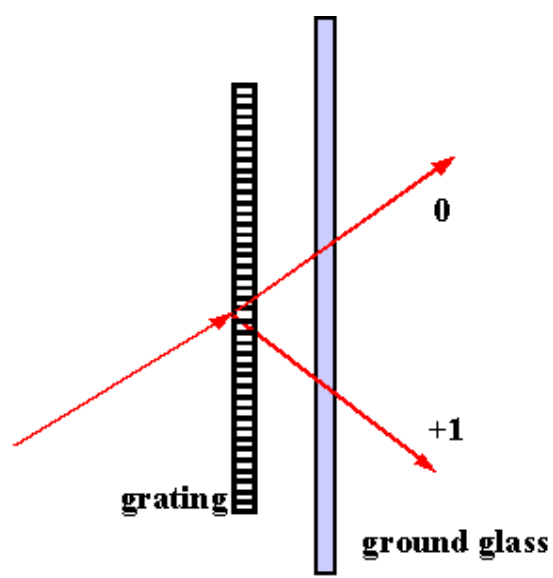

b

Fig.4. Propagation of the beams through the shearing device of holographic grating and ground glass: a) grating with smaller spatial frequency; b) grating with bigger spatial frequency 
The intensity of the additional orders is smaller but nevertheless they affect the contrast of the ESPSI fringes obtained. If the spatial frequency of the diffraction grating is high (Fig. 4b) the contrast is better as the two main orders only are projected onto the glass plate.

It was also observed that the size of the field of view strongly depends on the spatial frequency of the grating. When a high spatial frequency grating was used the field of view with good contrast of the ESPSI fringes is considerably decreased. We ascribe this observation to the fact that higher spatial frequency grating is characterised by higher angular selectivity and in this case only small part of the light scattered by the object surface satisfies the Bragg condition for reconstruction of the image in the +1 order. Taking into account the considerations above a $500 \mathrm{l} / \mathrm{mm}$ spatial frequency grating was chosen for the experiments in dynamic mode of loading of the object.

The ESPSI system described above with a grating 500 lines $/ \mathrm{mm}$ was used for studying the vibration modes of an edge clamped aluminium diaphragm (75 mm diameter, $0.5 \mathrm{~mm}$ thick). The diaphragm was excited at different frequencies in the range from $2000 \mathrm{~Hz}$ to $7000 \mathrm{~Hz}$ by a loudspeaker placed behind it. The loudspeaker is driven by a sinusoidal signal of amplitude $0.5 \mathrm{~V}$ or $1 \mathrm{~V}$. Time-averaged TV shearography was used for all vibration studies. In addition to the ESPSI experimental technique, theoretical predictions of resonant frequencies based on Ansys 8.1 package are also presented.

\section{RESULTS AND DISCUSSION}

The ESPSI system using holographic grating and a ground glass to shear the image was used to record different vibrating modes of the an edge clamped aluminium diaphragm (Fig. 5 and Fig. 6). A filter with a 3x3 window was used to reduce the speckle noise in the images. The experimental results show that the ESPSI system used is suitable for detection of the resonant frequencies of the vibrating aluminium diaphragm. The detection principle is based on the fact that clear fringe patterns with fringe contrast above $90 \%$ appear only at resonant frequencies. The introduction of different shear does not affect the mode shape.

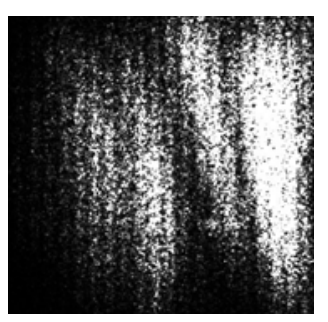

$2100 \mathrm{~Hz}$

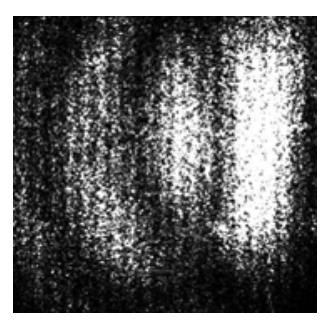

3800 Hz

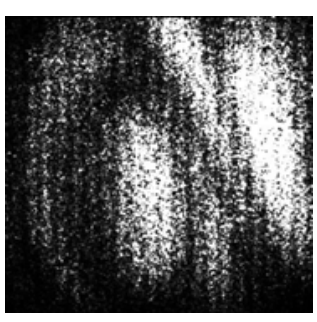

$5200 \mathrm{~Hz}$

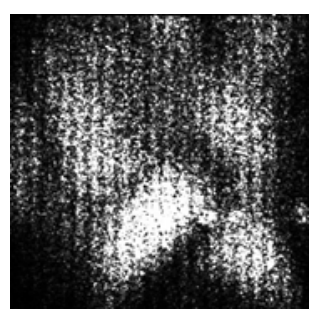

$6200 \mathrm{~Hz}$

Fig. 5. ESPSI vibration modes of a central part of an edge clamped aluminium diaphragm. The shear is $8 \mathrm{~mm}$. The amplitude of the sinusoidal signal driving the loudspeaker is $1 \mathrm{~V}$. The field of view is $23 \mathrm{~mm} \times 23 \mathrm{~mm}$ 


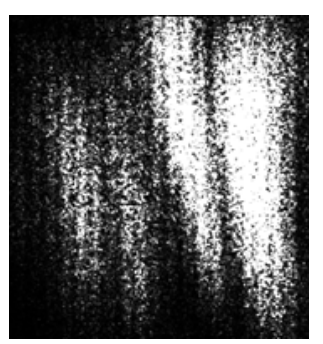

$2100 \mathrm{~Hz}$

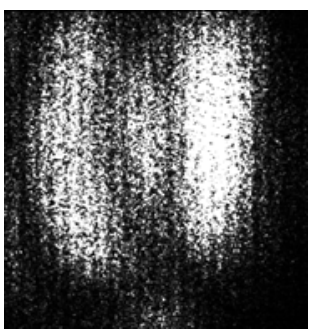

$3800 \mathrm{~Hz}$

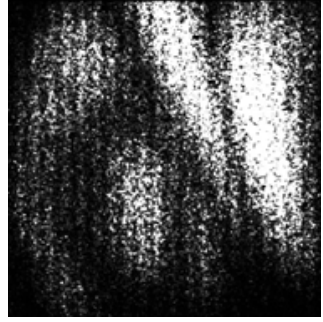

$5200 \mathrm{~Hz}$

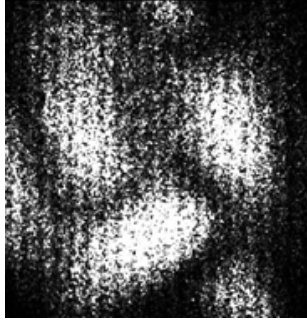

$6200 \mathrm{~Hz}$

Fig. 6. ESPSI vibration modes of a central part of an edge clamped aluminium diaphragm. The shear is $11 \mathrm{~mm}$. The amplitude of the sinusoidal signal driving the loudspeaker is $0.5 \mathrm{~V}$. The field of view is $23 \mathrm{~mm} \times 23 \mathrm{~mm}$

A modal analysis was carried out using the ANSYS finite element program. The aluminium disk was modelled using approximately 1100 linear shell elements (Fig. 7). This number was sufficient to resolve all required modes. Nodal position restraints were placed on all nodes at the circumference. This boundary condition most closely matches the actual restraint of the disk.

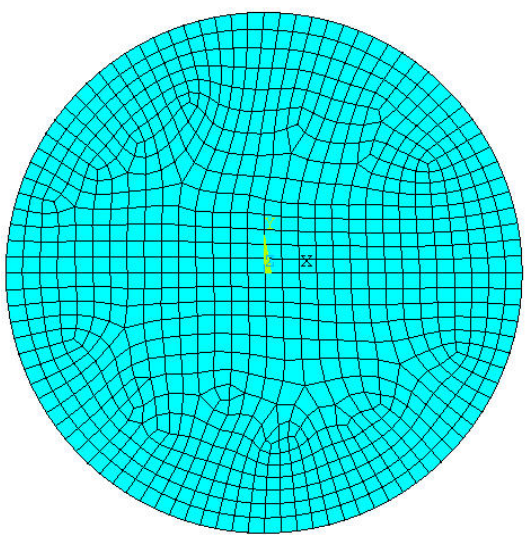

Fig. 7. The linear shell elements used in FEM of the aluminium disk

The comparison of the theoretically calculated and experimentally measured frequencies is presented in Table 1 . The differences between the predicted and measured values are within the experimental accuracy of the measurement.

Table 1. Comparison of theoretical predicted resonant frequencies with the experimental results

for a vibrating aluminium diaphragm

\begin{tabular}{|c|c|c|c|c|}
\hline Mode & $\mathbf{1}$ & $\mathbf{2}$ & $\mathbf{3}$ & $\mathbf{4}$ \\
\hline ESPSI, Hz & $2100 \pm 2 \%$ & $3800 \pm 1.5 \%$ & $5200 \pm 1 \%$ & $6200 \pm 1 \%$ \\
\hline FEM, Hz & $2256 \pm 7 \%$ & $3514 \pm 7 \%$ & $4994 \pm 7 \%$ & $6169 \pm 7 \%$ \\
\hline
\end{tabular}




\section{CONCLUSIONS}

It is demonstrated that the ESPSI systems using holographic optical elements to shear the image are simple and flexible and offer a simple way to introduce discrete shear steps between two images and thus to control the sensitivity of the system. Another advantage of these systems is that it is easy to change the shearing direction by rotating of the grating around its normal. Advantages include also the low cost of such a system and the potential to use large apertures.

The paper shows that the ESPSI systems using holographic optical elements can be used for the analysis of vibrating systems. High contrast interference patterns at characteristic frequencies of vibration of the object under study were recorded. A theoretical model of the vibrating object was generated using Ansys 8.1 Package. The theoretical results obtained for the resonant frequencies are in very good agreement with the resonant frequencies extracted from the experiments. The experimental results obtained are promising for future applications of these systems for modal analysis. Larger aperture HOEs are currently being prepared so that the potential for modal mapping can be fully assessed.

We therefore conclude that photopolymer HOEs can be used to make simple shearing systems, which can analyse and possibly map the modal behaviour of vibrating objects. The results indicate that the potential for such systems in full field modal analysis is significant.

\section{ACKNOWLEDGMENTS}

Acknowledgements are made to Technological Sector Research Programme Strand III supported by the Irish Government. Emilia Mihaylova and Izabela Naydenova would like to thank the Arnold F. Graves Scholar Programme and FOCAS at Dublin Institute of Technology.

\section{REFERENCES}

1. C. Joenathan C. and Torroba R., "Simple electronic speckle shearing pattern interferometer", Opt. Lett. 15 (20), 1159-1161 (1990).

2. R. S. Sirohi, "Speckle methods in experimental mechanics”, in Speckle Metrology, Ed. R. S. Sirohi, Mercel Dekker, New York (1993).

3. Y. M. He, C. J. Tay, H. M. Shang, "Digital phase-shifting shearography for slope measurement", Opt. Eng. 38 (9), 1586-1590 (1999).

4. H. M. Shang, Y. Y. Hung, W. D. Luo, F. Chen, “Surface profiling using shearography”, Opt. Eng. 39(1), 23-31 (2000).

5. P. Hariharan, “Speckle-shearing interferometry: a simple optical system”, Appl. Opt. 14 (11), 2563 (1975).

6. Y. Iwahashi, K. Iwata, and R. Nagata, “Single-aperture speckle shearing interferometry with a single grating”, Appl. Opt. 23 (2), 247-249 (1984). 
7. C. Joenathan and R. S. Sirohi, "Holographic gratings in speckle shearing interferometry”, Appl. Opt. 24 (17), 27502751 (1985).

8. H. Rabal, R. Henao, R. Torroba, "Digital speckle pattern shearing interferometry using diffraction gratings”, Optics Comm. 126, 191-196 (1996).

9. C. Joenathan, L. Bürkle, “Electronic speckle pattern shearing interferometer using holographic gratings”, Opt. Eng. 36 (9), 2473-2477 (1997).

10. S. Martin, P. Leclère, V. Toal, Y. Renotte and Y. Lion, "Characterisation of acrylamide-based photopolymer holographic recording material”, Optical Engineering, 32 (12), 3942 - 3946 (1994).

11. C. M. Vest, Holographic Interferometry (John Wiley, New York 1979).

12. Y. Y. Hung and C. Y. Liang, "Image shearing camera for direct measurement of surface-strains”, Appl. Opt. 10(7), 1046-1050 (1979).

13. Emilia Mihaylova, Izabela Naydenova, Suzanne Martin, Vincent Toal, "Electronic speckle pattern shearing interferometer with a photopolymer holographic grating”, Applied Optics 43 (12), 2439 - 2442 (2004). 